\title{
Sistema de expertos y la Contabilidad
}

\author{
Pérez Trejos, Leidy Karina \\ Corporación Universitaria de Ciencia y Desarrollo - Uniciencia, \\ Bucaramanga, Colombia \\ Universidad de Los Andes, Programa de Doctorado en \\ Ciencias Contables, Mérida, Venezuela \\ E-mail: leidyk.perez@unicienciabga.edu.co
}

Recibido: 25 noviembre de 2019 Aprobado: 22 diciembre de 2019

\begin{abstract}
Resumen
Para nadie es un secreto, que hoy las tecnologías de la información se han convertido en herramientas útiles e indispensables para las actividades propias de la industria, el comercio, la empresa, los negocios, y el desarrollo del trabajo. Este estudio busca aclarar conceptualmente qué es un sistema de expertos en la generalidad, y definirlo luego en el campo del sistema contable, asumiendo en profundidad la naturaleza, utilidad, y aplicaciones de este dominio del conocimiento, su experticia en el campo de la contabilidad. Haciéndolo importante, dado que la intrusión de los sistemas de expertos, ha sido de una gran utilidad, porque de la informatización estructurada, convencional de suministrar datos e información, se ha pasado a la del conocimiento, logrando mejorar la calidad de los trabajos profesionales y disminuyendo el tiempo de ejecución.
\end{abstract}

Palabras clave: Sistemas, Sistemas de expertos, Inteligencia artificial. 


\title{
Expert System and Accounting
}

\begin{abstract}
Abstrac
It is no secret to anyone, that today information technologies have become useful and indispensable tools for the activities of industry, commerce, business, business, and work development. This study seeks to clarify conceptually what a system of experts in generality is, and then define it in the field of the accounting system, assuming in depth the nature, utility, and applications of this domain of knowledge, its expertise in the field of accounting. Making it important, given that the intrusion of expert systems, has been very useful, because of the structured, conventional computerization of providing data and information, it has passed to that of knowledge, improving the quality of professional work and decreasing runtime
\end{abstract}

Keywords: Systems, Expert systems, Artificial intelligence.

\section{Introducción}

Para nadie es un secreto, que hoy las tecnologías de la información se han convertido en herramientas útiles e indispensables para las actividades propias de la industria, el comercio, la empresa, los negocios, y el desarrollo del trabajo. Como lo expresa Ceballos (2010) influyen de manera notable el hacer y el quehacer del hombre postmoderno, que navega en un mundo más complejo que el de la modernidad.

En lo precedente las tecnologías de la información se han dispuesto para afrontar el problema de la sostenibilidad de las empresas, fundado en la productividad y la competencia de tal modo, que todas las disciplinas, la información, y el conocimiento son afectados por ellas, y las organizaciones empresariales expresa Rivas (s. f) se ven incrementalmente, imbuidas en un entorno más cómodo aunque duro a la vez: cómodo puesto que existen las herramientas que facilitan todo tipo de trabajos, eliminando aquellas tareas más pesadas, ásperas y rutinarias; pero como lo expresa Cisneros (2008) también incidiendo duramente, ya que ésta instrumentalidad, opera de manera incisiva en el mundo laboral, generando nuevas formas de trabajar e incluso de administrar, requiriendo una nueva mentalidad si se les ha de aprovechar en su integralidad.

Es así, que las tecnologías de la información, se han hecho necesarias no sólo para el ámbito de la comunicación, propiciar datos, sino también para el del conocimiento, de los saberes, por lo que no extraña, interpretando a García (1991) que no sólo manipulen datos para hacerlos información, sino que también trabajen con conocimientos, experiencia y saber hacer.

Cuando se habla de saber hacer y conocimientos, se entra en el área de la Inteligencia Artificial, y los Sistemas Expertos que desarrollan esta labor (Amador, 1996, p.56). Estos sistemas, reconocen Maté y Pazos (1988) intentan simular las respuestas que daría un experto humano en un dominio de conocimiento determinado, por lo que pueden ayudar, aunque no sustituir, a estos expertos. Sus aplicaciones en las empresas son múltiples, siendo relevantes en el sector financiero e industrial por lo que se infiere su utilidad en el sistema contable.

Este estudio busca aclarar conceptualmente qué es un sistema de expertos en la generalidad, y definirlo luego en el campo del sistema contable, asumiendo en profundidad la naturaleza, utilidad, y aplicaciones de este dominio del conocimiento, su experticia en el 
campo de la contabilidad.

\section{Sistema de expertos}

Para entender la naturaleza del Sistema experto (SE), es necesario echar una mirada a la inteligencia artificia (IA), sin la cual el SE, no hubiera sido posible. Así, "la Inteligencia Artificial supone la potencialidad de la máquina para reproducir algunos de los componentes esenciales de la mente humana, como la capacidad de aprendizaje, la deducción, la inducción o la capacidad de construir -a través de la comprensión de mensajes visuales y auditivos una cierta concepción del mundo" (p.49) (García, 1991), y con Soto (1986) permitiendo resolver problemas no totalmente definidos y crear asociaciones entre conceptos y fenómenos que el ser humano es, en ocasiones, incapaz de concebir.

En lo anterior, se puede entrar a definir un Sistema Experto, que en palabras de Rodríguez Marín(1991) es como "un programa especializado en un dominio concreto de aplicación, que incluye el conocimiento que poseen los humanos en dicha materia y que, fundamentalmente, proporciona respuestas similares a las que daría una persona experta en el área”.

Aquí es importante aclarar que el Sistema de expertos, no maneja un conocimiento reglado, por no decir que matemático. El conocimiento no es preciso, pues obra sobre juicios, del qué hacer que es aproximado. Se entiende que se puede automatizar un Sistema de expertos desde los razonamientos heurísticos, es decir los aproximados, intuitivos, basados en asociaciones empíricas, los juicios surgidos del saber hacer de un individuo, como lo manifiesta Andreu (1991).
Un concepto relevante e integral, el de Jackson (1990) quien asume el SE, como un sistema de información basado en el conocimiento, que usa su conocimiento de un área de aplicación compleja y específica a fin de actuar como un consultor experto para los usuarios finales.

Infiere la anterior definición, que Los sistemas expertos facilitan respuestas sobre un problema específico de un área del conocimiento, a partir de inferencias, juicios similares a los que emiten los humanos sobre los conocimientos obtenidos en una base de conocimientos, naturalmente especializados.

Sin embargo, no sobra recordar que Los sistemas expertos forman parte de la ciencia de la computación y dentro de ésta se ubican en la rama de la inteligencia artificial; y que el poder de resolución de un problema en un programa de computadora viene del conocimiento de un dominio específico, no solo de las técnicas de programación y el formalismo que contiene (p.93) (Castillo, Gutiérrez and Hedi, 1998). Es decir que obra desde la forma y el contenido. Ofrece procedimientos que desarrollan la capacidad de la emisión de juicios.

\section{Características}

Un sistema experto, no es el resultado de lo gratuito. Su construcción no ha sido un proceso fácil, ya que para que un sistema actúe como un verdadero experto, es deseable que sea capaz de realizar procesos de razonamiento, por ello, atendiendo a Cuena (1995) debe reunir, en lo posible, lo más importante de las características de un experto humano, esto es: 0 apreciaciones.

- Solidez en el dominio de su conocimiento. 
- Habilidad para adquirir conocimiento.

- Fiabilidad, para poder confiar en sus resultados

- Capacidad para resolver problemas.

A pesar de las peculiaridades anteriores, algunos usuarios manifiestan desconfianza frente al sistema, si como lo aprecia Dudamel (2001), por la complejidad de los problemas que usualmente tiene que resolver un sistema experto, puede existir cierta duda en el usuario sobre la validez de respuesta obtenida

Pero prosigue Dudamel (2001), "por este motivo, es una condición indispensable que un sistema experto sea capaz de explicar su proceso de razonamiento o dar razón del por qué solicita tal o cual información o dato". (p.12) Se deduce, entonces que un SE, si se asimila a la naturaleza de un experto humano, debe al igual, ser capaz de explicar, es decir dar razón, valga lo tautológico, de sus propios razonamiento o juicios.

\section{Tipos de sistemas expertos y su relevancia}

Considera Fumanal (1991) que existen tres tipos de sistemas expertos:

- Basados en reglas: Aplicando reglas heurísticas apoyadas generalmente en lógica difusa para su evaluación y aplicación.

- Basados en casos CBR (Case Based Reasoning): Aplicando el razonamiento basado en casos, donde la solución a un problema similar planteado con anterioridad se adapta al nuevo problema.

- Basados en redes:Aplicandoredes bayesianas, basadas en estadística y el teorema de Bayes.

En lo precedente, surge la pregunta ¿por qué utilizar un sistema experto? A esta pregunta, responde Andreu (1991) con su ayuda, la del sistema experto, personas con poca experiencia pueden resolver problemas que requieren un conocimiento formal especializado. Se pueden obtener conclusiones y resolver problemas de forma más rápida que los expertos humanos.

Prosigue Andreu (1991), “estos sistemas razonan, pero en base a un conocimiento adquirido y no tienen sitio para la subjetividad. Se ha comprobado que tienen al menos, la misma competencia que un especialista humano." (p.88)

Considera Shoham (1993) que el uso del Sistema de expertos, es especialmente recomendado en las siguientes situaciones:

- Cuando los expertos humanos en una determinada materia son escasos.

- En situaciones complejas, donde la subjetividad humana puede llevar a conclusiones erróneas.

- Cuando es muy elevado el volumen de datos que ha de considerarse para obtener una conclusión.

\section{Ventajas}

Entre las ventajas que ofrece un sistema de expertos, reconocen Alonso y otros (1992), están:

- Producción y productividad mayores. Pueden trabajar más rápido que lo humanos. Están disponibles ininterrumpidamente de día y noche, ofreciendo siempre su 
máximo desempeño. Pueden duplicarse ilimitadamente, i.e. tener tantos de ellos como se requieran.

- Operación en entornos peligrosos. Muchas tareas requieren que los seres humanos operen en entornos hostiles y peligrosos.

- Captación de experiencia escasa y su dimensión. Uno de los principales beneficios de los sistemas expertos es su facilidad de trasmitir experiencia a través de fronteras internacionales.

- Siempre se ajustan a las normas establecidas y son consistentes en su desempeño, i.e. no desarrollan apreciaciones subjetivas, tendenciosas, irracionales o emocionales.

- Confiabilidad. Los sistemas expertos son confiables. No padece de olvido, fatiga, dolor o comete errores de cálculo.

- No requiere un sueldo, promociones, seguros médicos, incapacidades.

- Accesibilidad al conocimiento y escritorios de vida. Hacen accesible el conocimiento (y la información) a mucha gente en diversos lugares. Siempre están dispuestos a dar explicaciones, asistir o enseñar a la gente, así como a aprender.

- Pueden tener una vida de servicio ilimitada. Funciones incrementadas de otros sistemas expertos. La integración de un sistema experto, con otros sistemas expertos hacen que estos últimos se vuelvan más eficientes, los sistemas integrados abarcan más aplicaciones, trabajan más rápido y producen resultados de mayor calidad.
- Capacidad para trabajar con información incompleta o inconcreta. En contraste con los sistemas de cómputo convencionales, un sistema experto puede trabajar con información incompleta al igual que los expertos humanos.

- Impartición de capacitación. Es factible que un sistema experto facilite la capacitación. El personal nuevo que trabaja con un sistema experto se vuelve más experimentado. La fusión de explicación puede servir como un dispositivo de de enseñanza y de ese modo puede efectuar apuntes que tal vez se inserten en la base de conocimiento.

- Mejoramiento de las funciones para resolver problemas. Un sistema experto mejora la solución de problemas permitiendo la integración de juicios de expertos de primera línea en el análisis. De este modo, un sistema experto tiene las posibilidades resolver problemas cuyo enlace y conocimiento supera a los de cualquier individuo.

- Reducción del tiempo para la toma de decisiones. Con el empleo de la recomendación del sistema, un ser humano puede tomar decisiones mucho más rápido.

- Reducción del tiempo fuera de servicio. Muchos sistemas expertos operacionales se emplean para diagnosticar malos funcionamientos y prescribir reparaciones.

\section{Los sistemas de expertos en contabilidad}

\section{Generalidades introductorias}

Los ámbitos iniciales de la aplicación de Los sistemas expertos, una vez desarrollados como 
conocimiento especializado fueron: medicina, geología, química, ingeniería, etc. (Waterman, 1986; Harmon y King, 1988; Rauch-Hindin, 1989), para realizar tareas según Hayes, y otros (1983) en interpretación, predicción, diagnóstico, diseño, planificación, instrucción, control, etc.

Las actividades administrativas, financieras y contables, no habrían de quedar por fuera de la experiencia de los SE, y a estos campos del conocimiento, también se le han aplicado los Sistemas expertos, pues se realizan muchas de las tareas antes descritas y, además, éstas cumplen la mayoría de los requisitos que son necesarios para poder desarrollar un sistema experto (i.e., las tareas requieren conocimiento especializado, existen auténticos expertos en la materia, los expertos son escasos, la pericia necesita ser localizada en distintos lugares, la mayoría de las tareas requieren soluciones heurísticas, ...) (Waterman, 1986, pp. 127-134).

Pero es de aclarar, que no en todas las tareas que se realizan en el campo de la contabilidad y las finanzas es necesario utilizar los sistemas expertos. Por lo que, aprecia Sierra (1995) en las tareas de auditoría que están perfectamente estructuradas, son muy mecánicas y pueden expresarse en forma algorítmica (e.g., preparación de balances, cálculo de ratios, muestreo, circularización,...) se puede, y es conveniente, utilizar la informática convencional (e.g., programas informáticos normales, tratamientos de textos, bases de datos, ...);

Prosigue Sierra (1995) en las tareas que estén semiestructuradas se pueden utilizar los sistemas de ayuda a la decisión (e.g., hojas de cálculo, sistemas de consulta de archivos, sistemas de representación y análisis de datos, ...); reservándose los sistemas expertos para las tareas que estén muy poco o nada estructuradas, pues en este tipo de tareas se requiere mucho del juicio de un experto y se utilizan reglas heurísticas para llegar rápidamente a una solución, dado que el campo de soluciones puede ser muy amplio (Sánchez, 1993e).

\section{Áreas de aplicación del sistema de expertos en la contabilidad}

Para este estudio, lo más relevante es establecer una taxonomía de la clasificación de las áreas dónde aplicar el Sistema de expertos en la contabilidad. En apreciación de Anderson y Bernard, (1986), las áreas de aplicación estarían en:

- Auditoría: Análisis de la materialidad y del riesgo, evaluación del control interno, planificación de la auditoría, evaluación de la evidencia, análisis de cuentas concretas, formación de opinión, emisión del informe, auditoría interna, auditoría informática, etc.

- Contabilidad de costes y de gestión: Cálculo y asignación de costes, asignación de recursos escasos, control y análisis de desviaciones, planificación y control de gestión, diseño de sistemas de información de gestión, etc.

- Contabilidad financiera: regulación legal, normas y principios contables, recuperación y revisión analítica de registros contables, diseño de sistemas contables, imputación contable, consolidación de estados contables, etc.

- Análisis de estados financieros: Análisis patrimonial, financiero y económico de los 
estados contables, salud financiera de la empresa, cálculo e interpretación de ratios, cálculo y análisis de tendencias, etc.

- Planificación financiera e industria de los servicios financieros: Planificación financiera corporativa, planificación financiera personal, análisis de inversiones, gestión de tesorería, mercado de valores, seguros, banca, concesiones de crédito, etc.

\section{Sistemas Expertos en Auditoría}

\section{Características generales del dominio}

Como consecuencia de los grandes cambios producidos en las empresas por el avance tecnológico actual, manifiesta Sánchez (1994) el trabajo de auditoría se ha visto modificado considerablemente, caracterizándose básicamente por los siguientes rasgos:

Aumento creciente de las normas y procedimientos de auditoría; normas y procedimientos de auditoría cada vez más complejos; cambios en las normas de ética profesional, que exigen un mayor control y una mayor calidad en la realización de los trabajos de auditoría; mayor competición entre las empresas de auditoría, resultando, como consecuencia de ello, unos honorarios de auditoría más bajos; ofrecimiento al cliente de nuevos servicios (e.g., asesoramiento fiscal, informático, ...); desarrollo de nuevos tipos de auditoría (e.g., auditoría de gestión operativa, auditoría informática, auditoría medioambiental, ...). Todas estas circunstancias han hecho que la profesión de la auditoría sea cada vez más competitiva y, como consecuencia de ello, se haya visto forzada a recurrir a las nuevas técnicas y herramientas que facilita la tecnología de la información y la inteligencia artificial, para conseguir una información más relevante y oportuna que facilite a los auditores poder tomar decisiones de una forma rápida y aumentar, por tanto, la eficacia y el nivel de calidad de la auditoría.

En lo precedente, es oportuno, conceptuar sobre La auditoría financiera de cuentas o auditoría externa que se asume como:

La actividad, realizada por una persona cualificada e independiente, consistente en analizar, mediante la utilización de las técnicas de revisión y verificación idóneas, la información económico-financiera deducida de los documentos contables examinados, y que tiene como objeto la emisión de un informe dirigido a poner de manifiesto su opinión responsable sobre la fiabilidad de la citada información, a fin de que se pueda conocer y valorar dicha información por terceros (p.1) (R.D. 1636/1990, Reglamento de Auditoría de Cuentas).

Reconocida la naturaleza de la auditoría financiera, se puede entrar a hablar de Los subdominios o campos potenciales de la auditoría en los que se pueden aplicar los sistemas expertos son muy amplios y variados, abarcando prácticamente todas las tareas de las auditorías en las que se requiera la utilización del juicio profesional del auditor.

Por lo tanto, es conveniente establecer una clasificación. En una primera clasificación, según Sánchez (1991) las aplicaciones de sistemas expertos en auditoría se podrían 
clasificar atendiendo a estas tres categorías: (1) sistemas expertos en auditoría externa, (2) sistemas expertos en auditoría interna y (3) sistemas expertos en auditoría informática. Pero considera necesario Sánchez (1991) que dado que el campo de la auditoría externa es muy amplio, es conveniente realizar una subdivisión del mismo.

Una forma práctica de establecer una clasificación de los sistemas expertos en auditoría externa es utilizando las fases del proceso de auditoría. Jacob y Bailey (1989), basándose en un trabajo anterior de Felix y Kinney (1982), descomponen el proceso de decisión en auditoría en las siguientes fases:

a) Orientación - El auditor obtiene conocimientos sobre las operaciones del cliente y su entorno y hace una valoración preliminar del riesgo y de la materialidad;

b) Evaluación preliminar de los controles internos;

c) Planificación táctica de la auditoría;

d) Elección de un plan para la auditoría;

e) Pruebas de cumplimiento de los controles;

f) Evaluación de los controles internos, basada en los resultados de las pruebas de cumplimiento;

g) Revisión del plan de auditoría preliminar;

h) Elección de un plan revisado para la auditoría;

i) Realización de pruebas sustantivas;

j) Evaluación y agregación de los resultados;

k) Evaluación de la evidencia - Podría dar lugar a unas pruebas más exhaustivas o formar la base de la elección de la opinión por el auditor;

1) Elección de una opinión que clasifique los estados financieros del cliente;

m) Informe de auditoría.

Por lo tanto, basándonos en el análisis de la literatura sobre los sistemas expertos que se han desarrollado hasta la fecha, y teniendo en cuenta las fases del proceso de auditoría, vamos a establecer una clasificación de los sistemas expertos en el dominio de la auditoría.

Ahora bien, como no se han desarrollado sistemas expertos en todas las fases, los agruparemos siguiendo el orden de las fases más importantes en las que, además, se han desarrollado sistemas expertos. La clasificación establecida es la siguiente:

1. Sistemas expertos en auditoría externa.

2. Sistemas expertos en auditoría interna.

3. Sistemas expertos en auditoría informática.

\section{Aplicaciones de sistemas expertos en el dominio de la auditoría}

Se infiere que la rama de la contabilidad en la que se han desarrollado más sistemas expertos es la auditoría. Las razones de este desarrollo se deben, en parte, al interés y al apoyo prestado por las grandes multinacionales de la auditoría, que han desarrollado o financiado muchas de las investigaciones llevadas a cabo en esta materia.

\section{Sistemas expertos en contabilidad de costes}

\section{Características generales del dominio}

Al igual que ha ocurrido en auditoría, 
los cambios tecnológicos actuales (i.e., perfeccionamiento del tratamiento $\mathrm{y}$ comunicación de la información, aumento de la importancia de la información como fuente para la toma de decisiones, nuevas técnicas de gestión, nuevas tecnologías de la producción, ...) también han afectado a la contabilidad de costes y a la contabilidad de gestión. Como consecuencia de estos cambios, en la década de los ochenta empezaron a surgir voces críticas que ponían en tela de juicio la información que suministraba la contabilidad de costes tradicional, pues ésta no suministraba información relevante y oportuna para poder tomar decisiones en el entorno actual (Johnson y Kaplan, 1987).

Pero estas críticas han sido relevantes, ello es indudable, porque han servido para producir una revitalización del papel de la contabilidad de costes y de gestión, considerándose ahora una de las principales fuentes de información para las empresas.

Importante determinar la funcionalidad de la contabilidad de costes y de gestión, dado el apoyo que le da el Sistema de expertos. Para Kaplan (1987) la contabilidad de costes persigue principalmente los siguientes objetivos básicos: (1) calcular los costes de las funciones, actividades, secciones, etc. del proceso productivo de la empresa, (2) calcular el coste de los productos y servicios generados por la empresa, (3) calcular el coste de los productos vendidos y analizar los resultados analíticos, (4) valorar los activos derivados del proceso productivo, con el fin de confeccionar los estados contables anuales. Mientras, prosigue Kaplan, que la contabilidad de gestión, además de recoger los objetivos anteriormente citados, se centra principalmente en el proceso de racionalización, planificación y control de los costes, con el fin de suministrar información relevante y oportuna para apoyar la toma de decisiones empresariales.

Es así, que se infiere que la contabilidad de gestión obviamente deriva de la contabilidad de costes, como consecuencia de las mayores demandas de información de las empresas modernas para poder tomar decisiones.

Reconocida la naturaleza de la contabilidad de costes y de gestión, ya se pueden considerar los sistemas expertos en contabilidad de costes y en contabilidad de gestión como un solo subconjunto de la contabilidad.

Para Sánchez (1991) los subdominios o campos potenciales de la contabilidad de costes y de gestión en los que se pueden aplicar los sistemas expertos son muy amplios y variados, destacando como más significativos los siguientes: de recursos escasos, gestión de la función de compras y de aprovisionamiento, gestión de inventarios, gestión de recursos humanos; cálculo del coste de las órdenes de trabajo, planificación y control de la gestión, costes estándares y análisis de desviaciones, control presupuestario, análisis de productos y combinaciones de ventas, presentación de informes económicos y estadísticas de costes y resultados, contr ol de nuevas tecnologías de la producción, diseño de sistemas de información de contabilidad de gestión, ayuda para la determinación de los costes pertinentes para la toma de decisiones, justificación de las inversiones en nuevas tecnologías. etc. 
Aplicaciones de sistemas expertos en el dominio de la contabilidad de costes y de gestión.

Hasta la fecha existen muy pocas aplicaciones de sistemas expertos en el campo de la contabilidad de costes y de gestión, y la mayoría de ellas lo son solamente a nivel teórico (Akers et al., 1986; Bouche y Retour, 1986; Estrin, 1988; Shim y Rice, 1988; Böer, 1989a, 1989b; 1989c).

Por lo regular, opina Salzedo (2005) las aplicaciones desarrolladas en este dominio están compuestas por dos módulos: un módulo que realiza los cálculos por medio de la informática tradicional o con la ayuda de una herramienta de ayuda a la decisión (e.g., una hoja de cálculo electrónica, un programa estadístico, ...), y un módulo de sistema experto, que realiza los procesos de decisión de interpretación, comparación, diagnóstico, predicción etc.

Por otra parte, reconoce Salzedo (2005) como característica muy importante de estas aplicaciones es que son específicas para cada empresa y para cada tipo de problema dentro de la empresa.

Otra característica importante de estos sistemas, añade Salzedo, es su capacidad para leer archivos de datos y para enlazar con las bases de datos de la empresa, de forma que se pueda disponer de información rápida para poder tomar decisiones.

\section{Sistema de expertos en contabilidad financiera}

\section{Características del dominio}

La contabilidad financiera se enfoca en el manejo de los estados contables que dan cuenta sobre la renta y la riqueza de la empresa, para lo cual somete a tratamiento y análisis a cuantas transacciones se producen entre la citada unidad económica y el mundo exterior a ella (Riaño, 201, p. 26).

$\mathrm{Si}$ se observa a profundidad, La contabilidad financiera asume como objetivo principal la preparación de los estados financieros de la empresa, con el fin de suministrar la información que necesitan los distintos usuarios de la empresa sobre el resultado y el patrimonio de la empresa. Importante tener en cuenta de la contabilidad financiera la estrecha vinculación que tiene con la legislación mercantil y contable, ya que está regulada por una serie de normas y principios de contabilidad generalmente aceptados, emitidos por el estado y por entidades de emisión de normas de contabilidad de reconocido prestigio (e.g., la SEC, el FASB, la AECA), que garantizan la homogeneidad y fiabilidad de la información contable frente a terceros.

\section{Aplicaciones de sistemas expertos en el} dominio de la contabilidad financiera

En la contabilidad financiera, manifiesta Quintanar (2007), como subdominio de la contabilidad, es en el que se han desarrollado menos sistemas expertos. Amplía Quintanar (2007) las aplicaciones que se han puesto en función, se han centrado principalmente sobre los siguientes temas: normativa legal y principios contables, interpretación de la regulación contable, asesoramiento legal no fiscal, consolidación de estados contables, revisión analítica de los estados financieros, transacciones

Pérez Trejos, Leidy Karina 
en moneda extranjera, diseño de sistemas de información contable, imputación contable, entre otros.

\section{Sistemas expertos en análisis contable}

\section{Características generales del dominio}

De acuerdo con el profesor Urías, el Análisis de Estados Financieros, utilizando ciertas técnicas, trata de investigar y enjuiciar, a través de la información contable, cuáles han sido las causas y los efectos de la gestión de la empresa para llegar a su actual situación y, así, predecir, dentro de ciertos límites ..., cuál será su desarrollo en el futuro, para tomar decisiones consecuentes. (Urías, 1992) (p. 179).

El análisis de estados financieros se puede dividir en tres fases: (1) examen, (2) análisis e interpretación, y (3) prescripción. En la primera fase, se realiza un examen o revisión de los documentos contables (i.e., balance, cuenta de pérdidas y ganancias, memoria, estado de origen y aplicación de fondos, ...), utilizando para ello una serie de técnicas o procedimientos específicos, tales como: comparaciones de masas patrimoniales, porcentajes, números índices, ratios, tendencias, etc. A continuación, en la segunda fase, se analiza e interpreta la información obtenida en la etapa anterior, y se realiza un diagnóstico de la situación actual de la empresa y de la gestión realizada. Y, por último, en la tercera fase, se realizan unas predicciones $\mathrm{y}$ se indican los remedios o soluciones que se pueden aplicar con el fin de mejorar la situación futura. Además, todos estudios y análisis se suelen realizar desde tres ángulos diferentes: el análisis patrimonial, el análisis financiero, y el análisis económico. (Alvarez, 1990; Rivero
Romero, 1990; Rivero Torre, 1991; Urías, 1992).

Aplicaciones de sistemas expertos en el dominio del análisis de estados financieros

Las aplicaciones de sistemas expertos desarrolladas en el campo del análisis de estados financieros, atendiendo a Quintanar (2007) se componen de dos módulos. Uno que es $\mathrm{s}$ de carácter informático convencional, realiza los cálculos por medio de la informática tradicional, o con la ayuda de una hoja de cálculo electrónica, y un módulo de sistema experto, que desarrolla los procesos de análisis e interpretación de los datos y de emisión del informe (normalmente por medio de un procesador de textos). muestran algunos de estos sistemas.

\section{Sistemas expertos en planificación}

\section{Características generales del dominio}

Para nadie es extraño que el entorno financiero actual es muy complejo y ha sufrido importantes cambios en los últimos tiempos (e.g., inflación, integración de la economía a nivel mundial, liberalización de la economía y de los mercados financieros, gran variedad de productos financieros, aumento de la presión fiscal y cambio continuo de su legislación, ...). Reconoce Suárez (1991) que debido a esta complejidad, las empresas y los individuos demandan, cada vez más, servicios de asesoramiento financiero que les permitan llevar una adecuada política de inversiones y de planificación financiera.

Precisa Suárez (1991) en la Economía Financiera de la Empresa, hay una rama que se encarga específicamente de los problemas 
de inversión y financiación empresarial. Esta disciplina, conocida en el área anglosajona con el nombre de Financial Management, se suele denominar en España con el nombre de Dirección (Administración o Gestión) Financiera.

Al igual, dentro de esta última disciplina, existen otras ramas importantes, que se refieren a aspectos concretos, como, por ejemplo: el Análisis de Inversiones, o Presupuesto de Capital (Capital Budgeting) es su acepción más moderna, que trata en general todos los problemas relacionados con las inversiones empresariales; el Análisis Financiero, que trata de analizar los problemas de carácter financiero producidos como consecuencia de dichas inversiones, y la Planificación Financiera, que estudia la planificación a corto, medio y largo plazo de la financiación de las inversiones realizadas (Suárez, 1991).

En el área de la Planificación Financiera, la mayoría de los sistemas expertos se centran especialmente en resolver los problemas de esta disciplina, aunque también algún sistema relacionado con el Análisis Financiero y la Análisis de Inversiones, campos éstos que están estrechamente relacionados con la Planificación Financiera.

La planificación financiera empresarial es aquella parte de la planificación empresarial que trata de los aspectos financieros de la empresa, aunque sin olvidar sus aspectos no financieros (Suárez, 1991, p. 608).

Por otra parte, por analogía con la empresa, la planificación financiera también puede realizarse a nivel personal, dando lugar a la planificación financiera personal, que abarca, entre otras, las siguientes materias: planificación de inversiones, planificación fiscal, gestión de patrimonios, planificación de seguros, planificación de la jubilación, gestión de tesorería, gestión de deudas, etc.

Infiere lo precedente que planificación financiera, el análisis de financiero, y el análisis de inversiones requieren una metodología muy específica. Entre las materias, métodos o procedimientos que se utilizan en estas disciplinas hay que destacar, por ejemplo: los flujos netos de caja, las tasas de rendimiento interno, el plazo de recuperación de la inversión, la deflación de los valores actuales, los árboles de decisión, el análisis bayesiano, los modelos de simulación, la programación lineal y la programación dinámica, el análisis de sensibilidad, la valoración de activos, el coste del capital, el riesgo económico y financiero, análisis de balances y cálculo de ratios, presupuestos, etc. (Suárez, 1991; Fernández, 1991).

\section{Aplicaciones en el dominio del planificación y análisis financiero de sistemas expertos}

Para resolver las tareas citadas en el apartado anterior, desde hace ya bastante tiempo, se ha venido utilizando la programación convencional; sin embargo, considera Quintanar (2007) ésta no permite considerar un componente subjetivo muy importante: el juicio profesional del analista.

Para Quintanar (2007) este aspecto es difícil de cuantificar en modelos matemáticos, ya que se basa, la mayoría de las veces, en conocimientos heurísticos, exclusivos del experto, adquiridos por éste, personalmente, tras muchos años de experiencia al tratar de resolver 
de forma sucesiva un problema.

Se analiza, que la tecnología de los sistemas expertos es muy útil para este tipo de tareas, ya que permite la utilización del conocimiento heurístico y del buen saber hacer de los analistas y planificadores financieros.

Hablando de funcionalidad y partes, la mayoría de las aplicaciones de sistemas expertos desarrolladas en el campo del planificación y análisis financiero contemplan estos aspectos, $\mathrm{y}$, normalmente, están compuestos por dos módulos: (1) un módulo que realiza los cálculos por medio de la informática tradicional o cualquier otro tipo de herramienta de ayuda a la decisión (e.g., hoja de cálculo, sistemas de simulación, modelos de optimización, etc.), y (2) el módulo de sistema experto propiamente dicho, que realiza los procesos de análisis e interpretación de los datos y de emisión del informe (normalmente por medio de un procesador de textos).

También es importante resaltar que los sistemas expertos de planificación financiera y análisis financiero son los sistemas expertos que han alcanzado un alto nivel de desarrollo, contradiciendo la opinión de que los sistemas expertos se refieren a dominios muy pequeños; tal vez porque la naturaleza de la información financiera es cualitativa y muy formalizada, y existe un marco teórico bastante desarrollado que permite tratar con un amplio grupo de variables de una forma sistemática (Connell, 1987, p. 225).

\section{Conclusiones}

La importancia de un sistema de expertos, naturalmente mediados por la inteligencia artificial (IE), es tan relevante, que los ha puesto a la altura apropiarse del conocimiento, y resolver problemas desde el mismo conocimiento, que no es otra cosa que poner a emitir juicios $y$ razonamientos a una máquina, situada a la altura del experto humano. Es decir, ponerla a pensar, indudablemente, no por encima del hombre, quien le suministra las capacidades para hacerlo desde la inteligencia artificial, que es ir más allá de las computadoras estructuradas, dispuestas solamente para brindar información y proporcionar datos.

Los sistemas de expertos, que habían beneficiado con su capacidad para obrar desde el conocimiento, apoyando la industria química y otras áreas de los saberes, ahora se han dispuesto desde la experticia de la inteligencia artificial, para apoyar a la contabilidad, dado que se han implementado para las áreas de la administración, las organizaciones, empresas y el mundo de los negocios.

En el campo de la contabilidad, la intrusión de los sistemas de expertos, ha sido de una gran utilidad, porque de la informatización estructurada, convencional de suministrar datos e información, se ha pasado a la del conocimiento, por no decir de una epistemología, que facilita al experto de inteligencia artificial construido para la contabilidad con la capacidad de adquirir conocimiento, emitirlo, y tomar decisiones confiables, lo que no era dable con la informatización estructurada, que requería del experto humano.

Se concluye finalmente que con la implementación del sistema de expertos en el campo de la contabilidad se logran grandes logros, si se observan como ventajas, que mejora 
de la calidad de los trabajos profesionales y disminución del tiempo de ejecución de los mismos, por una parte; en tanto, en adelante el asesoramiento no sólo será menos tenso, más flexible, por el acompañamiento del Sistema de expertos, en la toma de decisiones.

Pero, es el conocimiento donde habrá más aportes, pues con estos sistemas inteligentes, habrá una comprensión más profunda del conocimiento de los expertos, dada la utilidad del experticio artificial, como herramienta pedagógica y de formación del personal, para transmitir el conocimiento de los expertos a los nuevos contables

\section{Referencias bibliográficas}

Akers, Michael D., Porter, Grover L., Blocher, Edward J. y Mister, William G.: "Expert Systems for Management Accountants". Management Accounting [EE.UU.], marzo, 1986, pp. 30- 34. Traducción: Ing. Luis Quintero

Alonso, G.; Becerril, J. L. y Valor, F. (1992). "Sistemas basados en el conocimiento: ¿Ficción o realidad? CHIP. Septiembre. Pág. 30-34.

Andreu, C. (1991), "Los expertos comparten el riesgo", Dirección y Progreso, $N^{\circ} 119$, Pág, 37-40,

Amador Hidalgo, L. (1996). Inteligencia Artificial y Sistemas Expertos. Universidad de Córdoba.

Castillo, E., Gutiérrez, J.M. and Hadi, A.S. (1997) Expert Systems and probabilistic Network Models. Springer Verlag, New
York. Versión castellana publicada por la Academia de Ingeniería (1998)

Ceballos, Luis M (2010) Las nuevas tecnologías y el mundo laboral. México: Efe,

Cisneros, R (2008) Las tecnologías de la información y sistema de expertos. Madrid: Edaf

Cuena, J (1995) Notas sobre modelos de razonamiento. Dpto. de I.A., Facultad de Informática, UPM

Dudamel, Álvaro (2001) Sistemas integrados. Buenos Aires: ED. B

Félix, E W. L. y Kinney, W. R.: "Research in the Auditor's Opinion Formulation Process: State of the Art". The Accounting Review, abril, 1982, pp. 245-271. Traducción J, Stanley

García Merino, J. M. (1991). "Sistemas expertos: Técnicas de informática avanzada", Dirección y Progreso. Septiembre Octubre $\mathrm{N}^{\circ} 1$ 19. Pág. 49-50.

Hayes, Roth, Frederick, Waterman, Donald (1983)"Building Expert Systems". Addison- Wesley Publishing Company, Reading, Massachusetts. Traducción de Merino.

Jacob, Varguese S. y Bailey, Andrew D. Jr.: "A decision process approach to expert systems in auditing". Incluido en L.F. PAU, J. MOTIWALLA, Y.H. PAO, y H.H. TEH [Eds.]: "Expert Systems in Economics, Banking and Management". North- Holland, Amsterdam, 1989pp. 157-166. Traducción: Moritos 
Jackson, P. (1990). Introduction to Expert Systems Addison-Wesley.

Johnson, R. thomas y Kaplan, Robert S. (1988). "La contabilidad de costes. Auge y caída de la contabilidad de gestión". Plaza y Janes. Barcelona.

Maté Hernández, J. 1. y Pazos Sierra, J. (1988). "Ingeniería del Conocimiento. Diseño, Construcción y Sistemas Expertos", Cettico. Córdoba (Argentina). Pág. 525.

Quintanar, T. (2007). Sistemas Expertos y sus aplicaciones. Pachuca de Soto: Universidad Autonóma del estado de Hidalgo

Rivas, F. (s.f.). sistemas de información. Fundamento de diseño de bases de datos. Recuperado el 23 de marzo de 2014, de Universidad de Granada: http://elvex.ugr. es/idbis/db/docs/ intro/ A \% $20 \mathrm{Sistemas} \% 20 \mathrm{de} \% 20$ Informaci $\% \mathrm{C} 3 \% \mathrm{~B} 3 \mathrm{n}$.pdf

Rodríguez Marín, P. (1991). “Sistemas Expertos en la gestión empresarial". Dirección y Progreso. Septiembre - Octubre $N^{\circ} 119$. Pág. 42

Fumanal, L. (1991). 'Sistemas expertos: un proyecto informático". Dirección y Progreso, Septiembre-Octubre. $\mathrm{N}^{\circ} 1$ 19. Pág. 9Sánchez Tomás, Antonio (1994): "Sistemas expertos en auditoría". Tesis doctoral. Universitat de València, Valencia,

Sánchez, T. (1991). sistemas expertos en contabilidad. Revista Técnica Contable,
Sánchez, A. (1994): "Sistemas expertos en auditoría". Tesis doctoral. Universitat de València, Valencia, 1994.

Soto, J. (1986). "Sistemas integrados de oficina", Dirección y Progreso. $\mathrm{N}^{\circ}$ 85. Enero Febrero. Pág. 48.

Shoham, Y. (1993). Artificial Intelligence Techniques in Prolog, Morgan Kaufmann,

Urías, J. (1992). Análisis de Estados Financieros. McGraw-Hill, Madrid, 1992. 